Research Article

Open Access

\title{
Can hENT1 Predict Survival in Locally Advanced Pancreatic Carcinoma Received Gemcitabine Monotherapy?
}

\author{
Hesham Tawfik ${ }^{1 \star}$, Sarah MF Ahmed ${ }^{1}$, Eiman Adel Hasby Saad ${ }^{2}$ and Wael Mansour Said ${ }^{1}$ \\ 'Departments of Clinical Oncology and Nuclear Medicine, Faculty of Medicine, Tanta University, Tanta, Egypt \\ 2 Department of Pathology, Faculty of Medicine, Tanta University, Tanta, Egypt
}

\section{Article Info}

*Corresponding author:
Hesham Tawfik
Departments of Clinical Oncology and
Nuclear Medicine
Faculty of Medicine
Tanta University
Tanta, Egypt
E-mail: hetawfik65@gmail.com

Received: October 22, 2018

Accepted: November 23, 2018

Published: November 30, 2018

Citation: Tawfik $\mathrm{H}$, Ahmed SMF, Hasby Saad EA, Said WM. Can hENT1 Predict Survival in Locally Advanced Pancreatic Carcinoma Received Gemcitabine Monotherapy?. Madridge J Oncogenesis. 2018; 2(1): 47-51. doi: $10.18689 /$ mjo- 1000108

Copyright: (c) 2018 The Author(s). This work is licensed under a Creative Commons Attribution 4.0 International License, which permits unrestricted use, distribution, and reproduction in any medium, provided the original work is properly cited.

Published by Madridge Publishers

\begin{abstract}
Background: Pancreatic cancer is a very aggressive disease. 5-year survival rates remain low at $10 \%$ to $30 \%$ even with adjuvant therapy, as most patients will succumb to the disease secondary to high rate of local and systemic recurrences. Gemcitabine is one of the drugs of choice for the adjuvant treatment for patients with pancreatic cancer. The drug is transported into pancreatic cancer cells primarily by human equilibrative nucleoside transporter 1 (hENT1).
\end{abstract}

Aim: The aim of this study was to assess and evaluate the predictive and prognostic value of hENT1 expression in advanced pancreatic cancer patients.

Methods: prospective and retrospective study was conducted on 30 patients presented with advanced pancreatic cancer; Immuno-histochemical staining of tumor tissue was performed for detecting hENT1 expression. All the patients have been treated with gemcitabine based chemotherapy.

Results: Sixty percent of patients had high hENT1 expression and $40 \%$ had low hENT1 expression. Overall survival after median follow up of 14 months showed that patients with high hENT1 expression had longer Overall survival than patients with low hENT1 expression and the difference was statistically significant $(p=0.001)$ and $(p=0.0025)$ respectively. Further, patients with high hENT1 expression showed longer disease free survival than patients with low hENT1 expression, and the difference was statistically significant $(p=0.002)$.

Conclusions: A high level of hENT1 expression is significantly associated with a longer survival in patients who received gemcitabine mono therapy. Hence, the study suggests hENT1 expression as potential assay for the prediction of response to gemcitabine based chemotherapy.

Keywords: Pancreatic cancer; hENT1; Gemcitabine; Survival; Marker.

\section{Introduction}

Pancreatic cancer (PC) representing the fourth leading cause of cancer-related mortality among US men and women and 10th cause of cancer -related death in Egypt $[1,2]$. Due to aggressiveness of the tumor, the diagnosis of PC is rarely made at an early stage. More than $90 \%$ of PC appears in the late stage of disease and only $10 \%$ of patients have respectable tumors at the time of diagnosis [3]. Many prognostic factors have been investigated thoroughly, especially from the histologic examination of the respected tumor $[4,5]$. 
Gemcitabine is the standard chemotherapy treatment for pancreatic cancer and up to $30 \%$ in general can be expected to respond to treatment [6]. Human equilibrative nucleoside transporter 1 (hENT1) proteins are trans-membrane glycoproteins that localized to the plasma membrane [7]. They are capable of mediating gemcitabine uptake in the direction of the concentration gradient, which lead for enhanced gemcitabine effects on the tumor cells [7].

Several studies suggested hENT1 evaluation has the strongest clinical support and a significant role as a predictive and prognostic marker with gemcitabine-based chemotherapy [7-12].

In this study we aim to evaluate the predictive and prognostic value of hENT1 in advanced pancreatic carcinoma.

Patients and Methods: This is a prospective and retrospective study conducted at clinical oncology and nuclear medicine department Tanta university hospitals from July 2012 to July 2017.

Inclusion Criteria: Advanced pancreatic carcinoma, age $>18 \&<70$ years, Performance scale $0-2$ according to ECGO score [13], adequate CBC, Liver and kidney function tests, all tissue biopsies taken either by $\mathrm{u} / \mathrm{s}$ guided biopsy or CT guided biopsy were formalin fixed and paraffin embedded, and no prior chemotherapy or radiotherapy treatment.

Exclusion Criteria: Patients with prior chemotherapy or radiotherapy treatment, pregnant or lactating mothers and those with active infection were excluded; also patients with previous history of hypersensitivity reactions or uncontrolled medical illness or other malignant disease were not enrolled in the study.

\section{Patients and Methods}

Patients received Gemicitabine (day 1,8,15 by $1000 \mathrm{mg} \backslash$ m2 IV over 30 minutes) repeat cycle every 28 days. Liver function test, creatinine and electrolyte were done every cycle and as clinically indicated. Imaging of the chest, abdomen and pelvis were checked every 3-6 months based on risk of recurrence and then as clinically indicated. Patients were assessed response at end of treatment and every 3 months using RECIST by Imaging of the chest, abdomen and pelvis every 3-6 months based on risk of recurrence and then as clinically indicated [14].

\section{Histopathology and Immunohistochemistry}

Tumor paraffin blocks were sectioned at $3 \mu \mathrm{m}$ thickness and stained for routine haematoxylin and eosin staining to confirm the diagnosis, then representative blocks were sectioned on charged slides. Staining was performed with the Dako Autostainer Link 48 (Dako). Before staining, sections were dried, deparaffinized and rehydrated followed by epitope retrieval at high pH (Dako PT Link machine, Dako). The automated staining procedure consisted of application of Envision Flex Peroxidase-Blocking Reagent (Dako) for 5 minutes, followed by incubation in first anti-hENT1 rabbit monoclonal antibody (clone SP120, dilution 1/100, Sigma-
Aldrich Co.) for 20 minutes, and then in a peroxidase-labeled polymer (Envision Flex/HRP; Dako) for 20 minutes, and finally application of the substrate chromogen [Substrate Working Solution (mix), Dako] for 10 minutes. After each step, the sections were rinsed in buffer ((Envision Flex wash buffer, Dako). After the final wash step, the slides were counterstained with haematoxylin, dehydrated, cleared and mounted.

\section{Immunohistochemical Evaluation}

The staining intensity for the hENT1 protein and the percentage of positive tumor cells were scored, and a composite score (hENT1 score) was obtained by calculating the sum of these two scores. The staining intensity for the hENT1 protein was assigned a score from 0 to 3 based on staining with $0+$ thus indicating no staining; $1+$, weakly positive; $2+$, moderately positive; and $3+$, strongly positive. The percentage of positive tumor cells was scored as follows: $0+$, no positive tumor cells; $1+,<50 \%$ positive cells; $2+, 50$ $80 \%$ positive cells; and $3+, \geq 81 \%$ positive cells. According to the hENT1 score, we classified tumors with the tumors with scores of $0-3$ as having a low hENT1 expression and tumors with scores of 4-6 as having a high hENT1 expression [9] (Figure 1 and Figure 2).

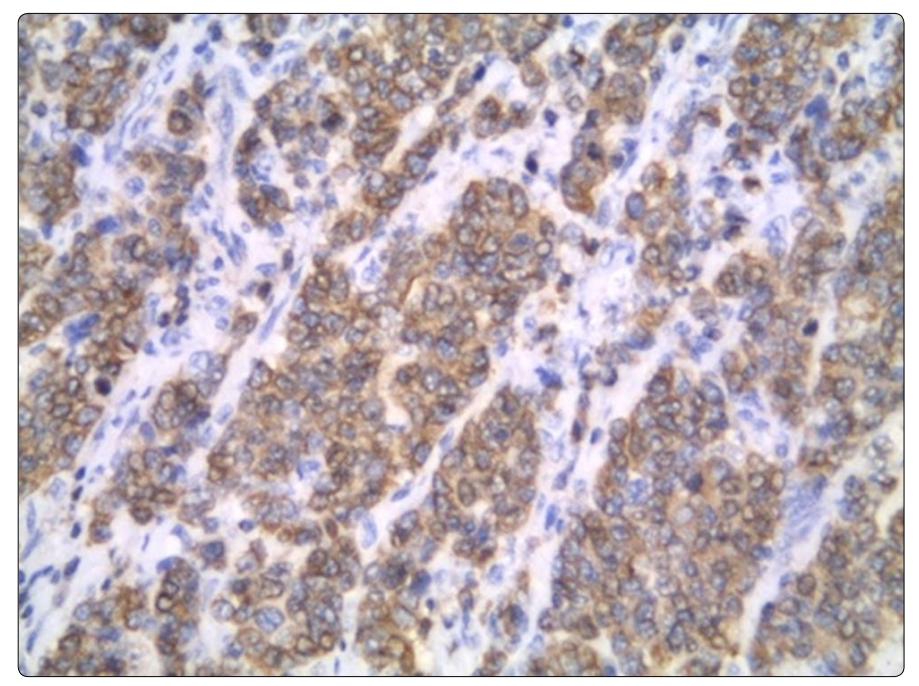

Figure 1. Pancreatic ductal carcinoma showing strong diffuse hENT1 expression [Immunoperoxidase X400]

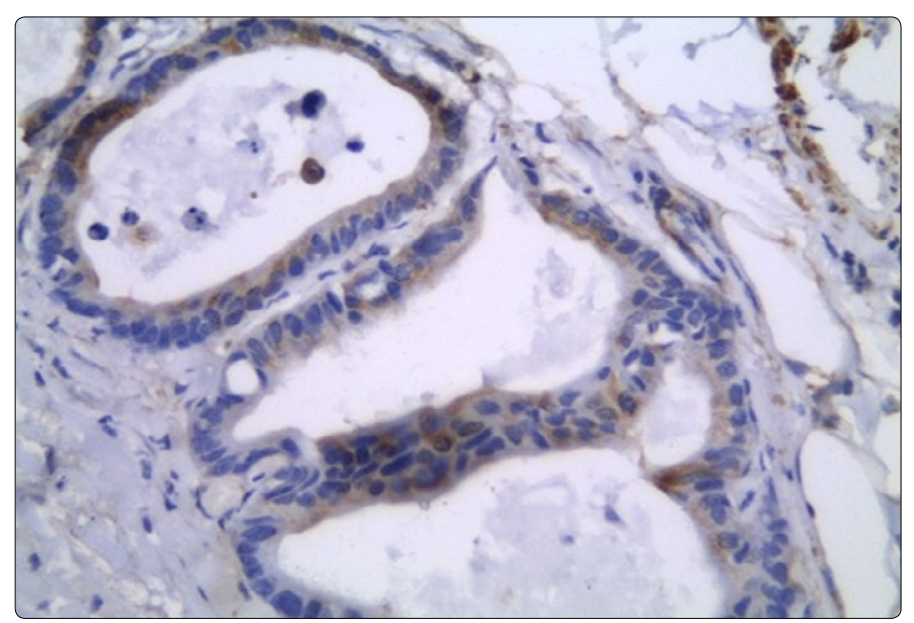

Figure 2. Well-differentiated pancreatic ductal carcinoma showing weak hENT1 intensity [Immunoperoxidase X400] 
The cumulative progression-free survival rate (the length of time during and after the treatment of a disease, such as cancer, that a patient lives with the disease but it does not get worse) and overall survival (he length of time from either the date of diagnosis or the start of treatment of cancer, that patients diagnosed with the disease are still alive) were estimated using the Kaplan-Meier method and were compared by the log-rank test. The predictors of outcome were assessed with the univariate analyses applying the Cox proportional hazard regression model. For all the statistical analyses, the level of significance was set at .05 . The SPSS statistical software program (SPSS for Windows 21.0J; SPSS Inc.; Chicago, IL) was used for all analyses.

\section{Results}

This study included thirty patients with advanced pancreatic carcinoma, most patients were male 17 (56.6\%), older than 50 years 17 (56.6\%), smoking history positive 16 (53.3\%), and 16 (53.3\%) were diabetic. Performance status was 1 in majority of patients 16(53.3\%), zero in 9(30\%), and were 2 in $5(16.7 \%)$.

The majority of patients suffered from pancreatic head tumor in 19(63.3\%), poorly differentiated carcinoma were found in $13(43 \%)$. High hENT1 levels expression in 18 (60\%) patients and low hENT1 levels were in 12 (40\%) patients shown in table 1.

Table 1. Patient's and tumor characteristics

\begin{tabular}{|c|c|c|}
\hline \multirow{2}{*}{ Factor } & \multicolumn{3}{|l|}{ Total no. of patients } \\
$\mathbf{n}=30$
\end{tabular}

In high hENT1 expression 18(60\%), most patients were less than 50 years $10(55.6 \%)$, males $13(72.2 \%)$, smokers $16(88.9 \%)$, diabetic $13(72.2 \%)$, head of pancreas tumors 14(77.8\%), tumor size $T_{2} 9(50 \%)$, moderate differentiated tumor 7(38.9\%), and were stage III 12(66.7\%). But none of these were statistically significant correlated with hENT1 expression.
Low hENT1 group $(n=12)$, most patients older than 50 years $9(50 \%)$, females $8(66.7 \%)$ with negative smoking history $(100 \%)$ and not diabetic (75\%). Most tumors were in body $(50 \%)$, T3 $(50 \%)$, and with poorly differentiated pathology (58.3\%), and were stage IV 10(83.3\%). also, after analysis of data all those factors were not significantly correlated with hENT1expression.

In high hENT1 expression group most patients showed PR 8(44.5\%), SD in 6(33.2\%), and PD (22.2\%). While in low hENT1 group most patients showed PD 5(41.7\%). Whereas SD in 4(33.3\%), and 3 patients showed PR 3(25\%), Reflecting a significant correlation between hENT1 expression and response rate to treatment with gemcitabine $(p=0.0331)$ shown in table 2.

Table 2. Comparison of clinico-pathological factors based on hENT1 score for all patients $(n=30)$

\begin{tabular}{|c|c|c|c|}
\hline \multirow{3}{*}{ Factor } & \multicolumn{2}{|c|}{ No. of patients (\%) } & \multirow{3}{*}{ P-value } \\
\hline & \multirow{2}{*}{$\begin{array}{l}\text { High hENT1 } \\
n=18 \text { (60\%) }\end{array}$} & \multirow{2}{*}{$\begin{array}{l}\text { Low hENT1 } \\
n=12(40 \%)\end{array}$} & \\
\hline & & & \\
\hline \multicolumn{4}{|l|}{ Age (years) } \\
\hline $\begin{array}{l}<50 \\
>50\end{array}$ & $\begin{array}{c}10(55.6 \%) \\
8(44.4 \%)\end{array}$ & $\begin{array}{l}3(25 \%) \\
9(75 \%)\end{array}$ & 0.271 \\
\hline \multicolumn{4}{|l|}{ Gender } \\
\hline $\begin{array}{l}\text { Male } \\
\text { Female }\end{array}$ & $\begin{array}{c}13(72.2 \%) \\
5(27.8 \%)\end{array}$ & $\begin{array}{l}4(33.3 \%) \\
8(66.7 \%)\end{array}$ & 0.147 \\
\hline \multicolumn{4}{|l|}{ Tumor location } \\
\hline $\begin{array}{c}\text { Head } \\
\text { Body } \\
\text { Tail }\end{array}$ & $\begin{array}{c}14(77.8 \%) \\
2(11.1 \%) \\
2(11.1 \%)\end{array}$ & $\begin{array}{c}5(41.7 \%) \\
6(50 \%) \\
1(8.3 \%)\end{array}$ & 0.289 \\
\hline \multicolumn{4}{|l|}{ Tumor size } \\
\hline $\begin{array}{l}\text { T2 } \\
\text { T3 } \\
\text { T4 }\end{array}$ & $\begin{array}{c}9(50 \%) \\
6(33.3 \%) \\
3(16.7 \%)\end{array}$ & $\begin{array}{c}1(8.3 \%) \\
6(50 \%) \\
5(41.7 \%)\end{array}$ & 0.0805 \\
\hline \multicolumn{4}{|l|}{ Grade } \\
\hline $\begin{array}{c}\text { Well } \\
\text { moderate } \\
\text { poorly }\end{array}$ & $\begin{array}{l}5(27.8 \%) \\
7(38.9 \%) \\
6(33.3 \%)\end{array}$ & $\begin{array}{c}2(16.7 \%) \\
3(25 \%) \\
7(58.3 \%)\end{array}$ & 0.1039 \\
\hline \multicolumn{4}{|l|}{ Smoking } \\
\hline $\begin{array}{l}\text { Positive } \\
\text { Negative }\end{array}$ & $\begin{array}{c}16(88.9 \%) \\
2(11.1 \%)\end{array}$ & $\begin{array}{c}0(0 \%) \\
12(100 \%)\end{array}$ & 0.109 \\
\hline \multicolumn{4}{|l|}{ DM } \\
\hline $\begin{array}{l}\text { Positive } \\
\text { Negative }\end{array}$ & $\begin{array}{c}13(72.2 \%) \\
5(27.8 \%)\end{array}$ & $\begin{array}{l}3(25 \%) \\
9(75 \%)\end{array}$ & 0.218 \\
\hline \multicolumn{4}{|c|}{ Clinical response } \\
\hline $\begin{array}{l}\text { PR } \\
\text { PD } \\
\text { SD }\end{array}$ & $\begin{array}{l}8 \text { (44.4\%) } \\
7(38.9 \%) \\
3(16.7 \%)\end{array}$ & $\begin{array}{c}3(25 \%) \\
5(41.7 \%) \\
4(33.3 \%)\end{array}$ & 0.0331 \\
\hline \multicolumn{4}{|l|}{ Stage } \\
\hline $\begin{array}{l}\text { III } \\
\text { IV }\end{array}$ & $\begin{array}{c}12(66.7 \%) \\
6(33.3 \%)\end{array}$ & $\begin{array}{c}2(16.7 \%) \\
10(83.3 \%)\end{array}$ & 0.118 \\
\hline
\end{tabular}

The median overall survival was 4 months $(95 \% \mathrm{Cl}$, 2.8685.132) for patients with low hENT1 expression and 9.5 months $(95 \% \mathrm{Cl}, 8.702-10.298)$ for patients with high hENT1 expression (HR for death 0.470; $95 \% \mathrm{Cl}$, 8.079-9.921; $\mathrm{p}=$ 0.001 ) which point to a significant positive correlation between hENT1 expression and overall survival in graph 1 . 


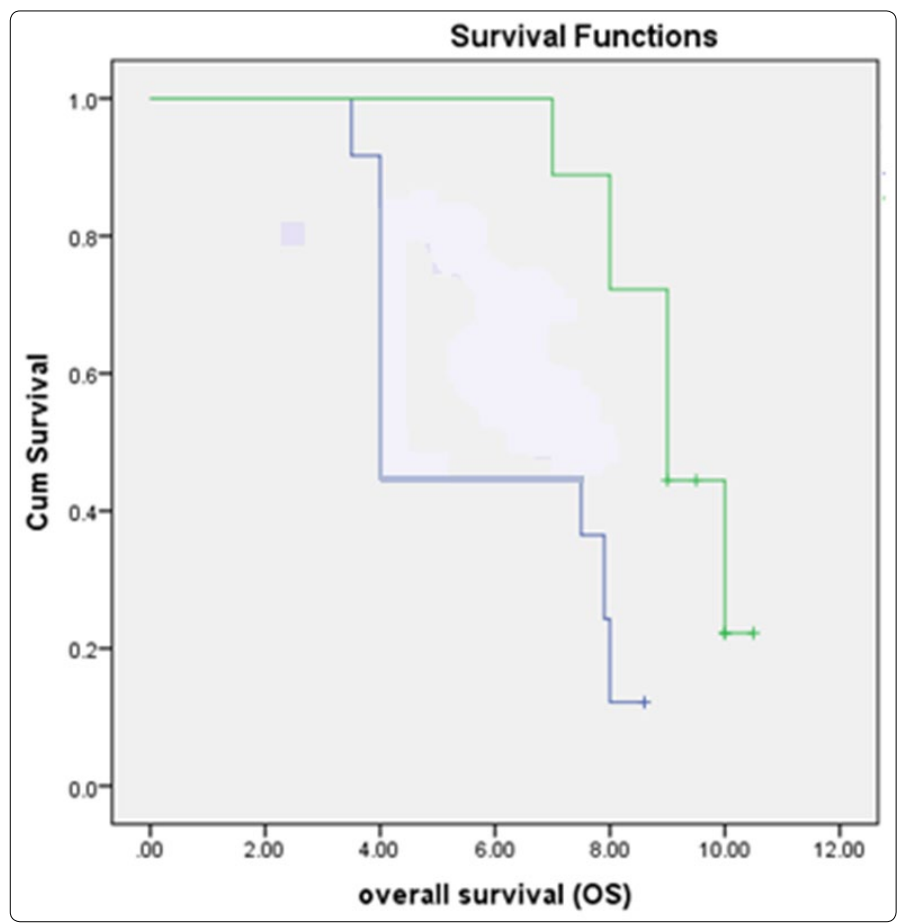

Graph 1. hENT1 score and overall survival of high and low hENT1 patients (0:3 represent low score (blue curve)) / (4:6 represent high score (green curve))

Results presented graph 2 shows a survival plot which revealed that the median PFS was 2.3 months $(95 \% \mathrm{Cl}, 1.767$ -2.833) for patients with low hENT1 expression and 4.5 months $(95 \% \mathrm{Cl}, 4.010-4.990)$ for patients with high hENT1 expression (HR for disease recurrence, $0.235 ; 95 \% \mathrm{Cl}, 2.540-3.460 ; p=0.002$ ). Indicating a significant positive correlation between hENT1 expression and progression free survival for the tested patients.

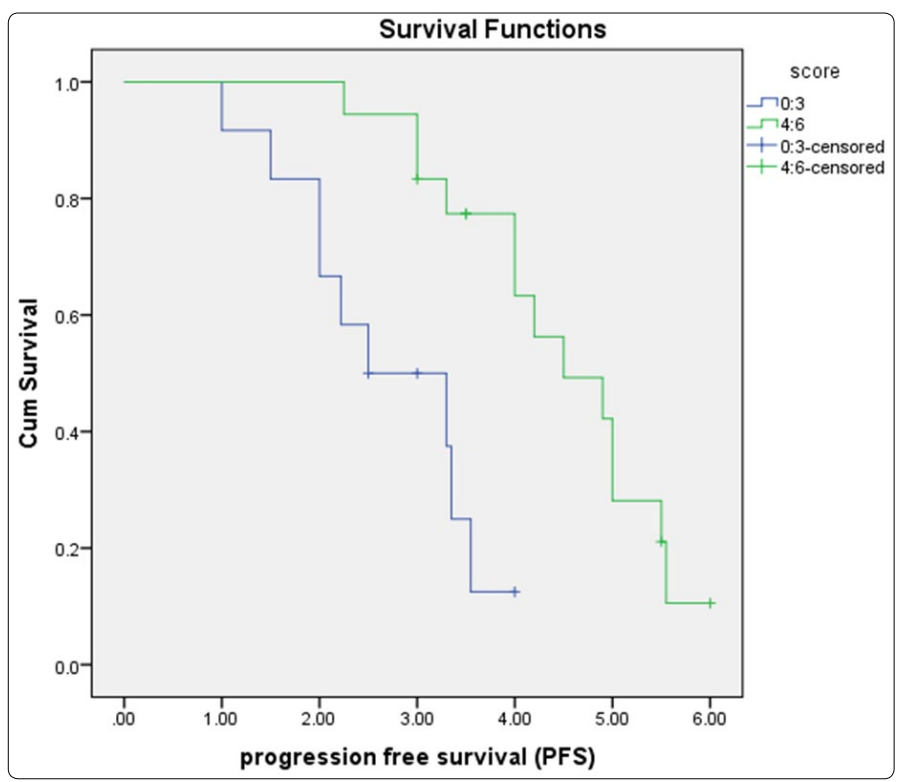

Graph 2: hENT1 score and progression survival of high and low hENT1 patients ( $0: 3$ represent low score (blue curve)) / (4:6 represent high score (green curve))

\section{Discussion}

In the present study we conducted a statistical analysis to evaluate the effect of some patient's characters on hENT1 expression for all the enrolled patients.
Assessment of hENT1 expression in the present study revealed that 18 patients were high hENT1 while 12 patients were low hENT1.Our findings matched with the results reported by Greenhalf et al [11] who surveyed hENT1 expression in patients treated with gemcitabine-based chemotherapy and showed longer PFS and OS in patients with high hENT1 expression than patients with low hENT1 expression.

On the other hand, Spratlin et al [7] reported somehow different results for study conducted on 21 patients where they found that 9 patients showed high hENT1 expression and 12 patients showed low hENT1 expression. They suggested that the results obtained maybe caused by a bias due to limited patients enrolled in the study.

The statistical analysis to evaluate the effect of some patient's characters on hENT1 expression for all the enrolled patients, could not find any evidences suggesting any significant variations in hENT1 expressions between all tested characters. Our results agree with previous reports by Marechal et al. [15].

In the present study, our results showed a strong significant positive correlation between hENT1 protein expressions and the overall survival and progression-free survival, patients with a high level of tumor hENT1 had a longer disease-free survival and overall survival when compared with patients with low tumor expression of hENT1.

Our results are in line with the results of previous studies assessing the prognostic and/or predictive value of hENT1 in pancreatic cancer Yamada et al. [10], Morinaga et al. [9], Giovannetti et al. [16], Marechal et al. [15]. Retrospective studies on patients with various stages of pancreatic cancer treated with gemcitabine showed pancreatic cancer with higher expression of hENT1 to have improved clinical outcomes after gemcitabine chemotherapy Giovannetti et al. 2006 [16], Spratlin et al. 2004 [7].

They attributed the improved overall survival and progression-free survival in patients with high hENT1 expression to the more efficient gemcitabine uptake in the tumor cells.

\section{References}

1. Ferlay J, Soerjomataram I, Ervik M, et al. GLOBOCAN 2012: v1.0, Cancer Incidence, Mortality and Prevalence Worldwide in 2012v1.0. IARC CancerBase No. 11. International Agency for Research on Cancer.

2. Amal S. Ibrahim, Hussein M. Khaled, Nabiel NH Mikhail, Hoda Baraka, Hossam Kamel. Cancer Incidence in Egypt: Results of the National Population-Based Cancer Registry Program. Journal of Cancer Epidemiology. 2014; Article ID 437971: 18. doi: 10.1155/2014/437971

3. Sharma C, Eltawil KM, Renfrew PD, Walsh MJ, Molinari M. Advances in diagnosis, treatment and palliation of pancreatic carcinoma: 1990-2010. World Journal of Gastroenterol. 2011; 17(7): 867-897. doi: 10.3748/wjg v17.i7.867

4. Bilici A. Prognostic factors related with survival in patients with pancreatic adenocarcinoma. World Journal of Gastroenterol. 2014; 20(31): 10802-10812. doi: 10.3748/wjg.v20.i31.10802

5. Franko J, Hugec V, Lopes TL, Goldman CD. Survival among pancreaticoduodenectomy patients treated for pancreatic head cancer < 1 or 2 cm. Ann Surg Oncol. 2013; 20(2): 357-361. doi: 10.1245/s10434012-2621-y 
6. Andriulli A, Festa $V$, Botteri $E$, et al. Neoadjuvant/preoperative gemcitabine for patients with localized pancreatic cancer: a meta-analysis of prospective studies. Ann Surg Oncol. 2012; 19(5): 1644-1662. doi: 10.1245/s10434-0112110-8

7. Spratlin J, Sangha R, Glubrecht D, et al. The absence of human equilibrative nucleoside transporter 1 is associated with reduced survival in patients with gemcitabine-treated pancreas adenocarcinoma. Clin Cancer Res. 2004; 10(20): 6956-6961. doi: 10.1158/1078-0432.CCR-04-0224

8. Fujita $\mathrm{H}$, Ohuchida $\mathrm{K}$, Mizumoto K, et al. Gene expression levels as predictive markers of outcome in pancreatic cancer after gemcitabine-based adjuvant chemotherapy. Neoplasia. 2010; 12(10): 807-817.

9. Morinaga S, Nakamura $Y$, Watanabe T, et al. Immunohistochemical analysis of human equilibrative nucleoside transporter-1 (hENT1) predicts survival in resected pancreatic cancer patients treated with adjuvant gemcitabine monotherapy. Ann Surg Oncol. 2012; 3:S558-64. doi: 10.1245/s10434-011-2054-z

10. Yamada S, Fujii T, Yabusaki N, et al. Clinical Implication of InflammationBased Prognostic Score in Pancreatic Cancer: Glasgow Prognostic Score Is the Most Reliable Parameter. Medicine (Baltimore). 2016; 95(18): e3582. doi: 10.1097/MD.0000000000003582
11. Grocock $C$, Harcus $M$, Harcus $M$, Neoptolemos J. Screening of High-Risk Families for Pancreatic Cancer. Pancreatology. 2009; 9: 215-222. doi: $10.1159 / 000210262$

12. Farrell JJ, Elsaleh $\mathrm{H}$, Garcia $\mathrm{M}$, et al. Human equilibrative nucleoside transporter 1 levels predict response to gemcitabine in patients with pancreatic cancer. Gastroenterology. 2009; 136(1): 187-195. doi: 10.1053/j.gastro.2008.09.067

13. Oken MM, Creech RH, Tormey DC, et al. Toxicity and response criteria of the Eastern Cooperative Oncology Group. Am J Clin Oncol. 1982; 5(6): 649655.

14. Eisenhauer, E.A., Therasse, P., Bogaerts, J, et al. New response evaluation criteria in solid tumours: revised RECIST guideline (version 1.1). Eur $J$ Cancer. 2009; 45(2): 228-247. doi: 10.1016/j.ejca.2008.10.026

15. Marechal, R., Mackey, J.R., Lai, R, et al. Deoxycitidine kinase is associated with prolonged survival after adjuvant gemcitabine for resected pancreatic adenocarcinoma. Cancer. 2010; 116(22): 5200-5206. doi: 10.1002/cncr.25303

16. Giovannetti E, Del Tacca M, Mey V, et al. Transcription analysis of human equilibrative nucleoside transporter-1 predicts survival in pancreas cancer patients treated with gemcitabine. Cancer Res. 66(7), 3928-3935 doi:10.1158/0008-5472.CAN-05-4203 